\title{
An optimal condition for the uniqueness of a periodic solutionfor systems of higher order linear functional differential equations
}

\author{
O. Kuzmych, S. Mukhigulashvili, and B. Pưz̆a
}




\title{
AN OPTIMAL CONDITION FOR THE UNIQUENESS OF A PERIODIC SOLUTION FOR SYSTEMS OF HIGHER ORDER LINEAR FUNCTIONAL DIFFERENTIAL EQUATIONS
}

\author{
O. KUZMYCH, S. MUKHIGULASHVILI, AND B. PŮŽA
}

Received 18th August, 2010

Abstract. Unimprovable efficient conditions are established for the unique solvability of the periodic problem

$$
\begin{aligned}
& u_{i}^{\left(p_{i}\right)}(t)=\sum_{j=2}^{i+1} \ell_{i, j}\left(u_{j}\right)(t)+q_{i}(t) \quad \text { for } 1 \leq i \leq n-1, \\
& u_{n}^{\left(p_{n}\right)}(t)=\sum_{j=1}^{n} \ell_{n, j}\left(u_{j}\right)(t)+q_{n}(t), \\
& u_{j}^{(p)}(0)=u_{j}^{(p)}(\omega) \quad \text { for } \quad 1 \leq j \leq n, 0 \leq p \leq p_{j}-1,
\end{aligned}
$$

where $\omega>0, n \geq 2, p_{j} \in \mathbb{N}, \ell_{i j}: C([0, \omega]) \rightarrow L([0, \omega])$ are linear bounded operators, and $q_{i} \in L([0, \omega])$.

2000 Mathematics Subject Classification: 34K06, 34K10

Keywords: Higher order linear functional differential system, periodic boundary value problem, uniqueness

1. STATEMENT OF PROBLEM AND FORMULATION OF MAIN RESUltS

On $[0, \omega]$, consider the system

$$
\begin{aligned}
& u_{i}^{\left(p_{i}\right)}(t)=\sum_{j=2}^{i+1} \ell_{i, j}\left(u_{j}\right)(t)+q_{i}(t) \quad \text { for } \quad 1 \leq i \leq n-1, \\
& u_{n}^{\left(p_{n}\right)}(t)=\sum_{j=1}^{n} \ell_{n, j}\left(u_{j}\right)(t)+q_{n}(t),
\end{aligned}
$$

Supported in part by the Academy of Sciences of the Czech Republic through the Institutional Research Plan No. AV0Z10190503.

(c) 2010 Miskolc University Press 
with the periodic boundary conditions

$$
u_{j}^{(p)}(0)=u_{j}^{(p)}(\omega) \quad \text { for } \quad 1 \leq j \leq n, 0 \leq p \leq p_{j}-1,
$$

where $\omega>0, n \geq 2, p_{j} \in \mathbb{N}, \ell_{i j}: C([0, \omega]) \rightarrow L([0, \omega])$ are the linear bounded operators, and $q_{i} \in L([0, \omega])$.

By a solution of the problem (1.1), (1.2) we understand a vector function $u=$ $\left(u_{i}\right)_{i=1}^{n}$ with $u_{i} \in \widetilde{C}^{p_{i}-1}([0, \omega])(i=\overline{1, n})$ which satisfies system (1.1) almost everywhere on $[0, \omega]$ and satisfies conditions (1.2).

Much work had been carried out on the investigation of the existence and uniqueness of the solution for a periodic boundary value problem for systems of ordinary differential equations and many interesting results have been obtained (see, for instance, $[1,2,5,6,13,14]$ and the references therein). However, an analogous problem for functional differential equations remains investigated in significantly less detail even for linear equations. In the present paper, we study problem (1.1) (1.2) under the assumption that $\ell_{n, 1}, \ell_{i, i+1}(i=\overline{1, n-1})$ are monotone linear operators. We establish new unimprovable integral conditions sufficient for unique solvability of the problem (1.1), (1.2) which generalize the well-known results of A. Lasota and Z. Opial (see Remark 1.2) obtained for ordinary differential equations in [7] and, on the other hand, extend our results obtained for linear functional differential equations in $[8,9,11]$. These results are also new if $(1.1)$ is the system of ordinary differential equations of the form

$$
\begin{aligned}
& u_{i}^{\left(p_{i}\right)}(t)=\sum_{j=2}^{i+1} l_{i, j}(t) u_{j}(t)+q_{i}(t) \quad \text { for } \quad 1 \leq i \leq n-1, \\
& u_{n}^{\left(p_{n}\right)}(t)=\sum_{j=1}^{n} l_{n, j}(t) u_{j}(t)+q_{n}(t),
\end{aligned}
$$

where $q_{i}, l_{i, j} \in L([0, \omega])$. The method used for the investigation of the problem considered is based on that developed in our previous papers [8-11] for functional differential equations.

The following notation is used throughout the paper: $\mathbb{N}($ resp., $\mathbb{R})$ is the set of all the natural (resp., real) numbers; $\mathbb{R}^{n}$ is the space of $n$-dimensional column vectors $x=\left(x_{i}\right)_{i=1}^{n}$ with elements $x_{i} \in \mathbb{R}(i=\overline{1, n}) ; \mathbb{R}_{+}=[0,+\infty[; C([0, \omega])$ is the Banach space of continuous functions $u:[0, \omega] \rightarrow \mathbb{R}$ with the norm $\|u\|_{C}=\max \{|u(t)|: 0 \leq$ $t \leq \omega\} ; \widetilde{C}^{p}([0, \omega])(p \in \mathbb{N})$ is the set of functions $u:[0, \omega] \rightarrow \mathbb{R}$ which are absolutely continuous together with their derivatives up to order $k$ inclusive. $L([0, \omega])$ is the Banach space of the Lebesgue integrable functions $p:[0, \omega] \rightarrow \mathbb{R}$ with the norm $\|p\|_{L}=\int_{0}^{\omega}|p(s)| d s$. If $\ell: C([0, \omega]) \rightarrow L([0, \omega])$ is a linear operator, then $\|\ell\|=$ $\sup _{0<\|x\|_{C} \leq 1}\|\ell(x)\|_{L}$. 
Furthermore, define the functional $\Delta: C([0, \omega]) \rightarrow \mathbb{R}_{+}$by setting

$$
\Delta(x)=\max \{x(t): t \in[0, \omega]\}-\min \{x(t): t \in[0, \omega]\} .
$$

Definition 1.1. We will say that a linear operator $\ell: C([0, \omega]) \rightarrow L([0, \omega])$ is nonnegative ( resp., nonpositive), if for any nonnegative $x \in C([0, \omega])$ the inequality $\ell(x)(t) \geq 0$ (resp., $\ell(x)(t) \leq 0$ ) for is satisfied $0 \leq t \leq \omega$. We will say that an operator $\ell$ is monotone if it is either nonnegative or nonpositive.

The following notation is used in the sequel:

$$
\begin{gathered}
B_{0}=1, \quad B_{1}=\frac{1}{15}, \quad B_{j}=B_{1} \sum_{m_{1}=1}^{2} \sum_{m_{2}=1}^{m_{1}+1} \ldots \sum_{m_{j-1}=1}^{m_{j-2}+1} \frac{1}{\eta\left(m_{1}\right) \ldots \eta\left(m_{j-1}\right)}, \\
C_{1}=\frac{1}{8}, \quad C_{j}=B_{1} \sum_{m_{1}=1}^{2} \sum_{m_{2}=1}^{m_{1}+1} \ldots \sum_{m_{j-1}=1}^{m_{j-2}+1} \frac{1}{\eta\left(m_{1}\right) \ldots \eta\left(m_{j-1}\right)} \prod_{i=1}^{m_{j-1}+1}\left(1+\frac{1}{2 i}\right),
\end{gathered}
$$

for $j \geq 2$, where

$$
\eta(t)=(2 t+1)(2 t+3)
$$

Let

$$
d_{0}=1, \quad d_{1}=4, \quad d_{2}=32, \quad d_{3}=192,
$$

and for $p \in \mathbb{N}$ put

$$
\begin{aligned}
d_{2 p+2} & =\frac{1}{\max \left\{\left(h_{p}(t) h_{p}(1-t)\right)^{1 / 2}: 0 \leq t \leq 1\right\}}, \\
d_{2 p+3} & =\frac{1}{\max \left\{\left(f_{p}(s, t) f_{p}(1-s, 1-t)\right)^{1 / 2}: 0 \leq s \leq 1,0 \leq t \leq 1\right\}},
\end{aligned}
$$

where the functions $f_{p}:[0,1] \times[0,1] \rightarrow \mathbb{R}_{+}$and $h_{p}:[0,1] \rightarrow \mathbb{R}_{+}$are defined as follows:

$$
f_{p}(s, t)=\sum_{j=0}^{p-1} \alpha_{p j} t^{2(j+1)}+\alpha_{p p} t^{2 p+3} s, \quad h_{p}(t)=\sum_{j=0}^{p} \beta_{p j} t^{2(j+1)},
$$

and

$$
\begin{gathered}
\alpha_{p j}=\frac{B_{j}}{3 \cdot 4^{j+1} d_{2(p-j)+1}}, \quad \beta_{p j}=\frac{B_{j}}{3 \cdot 4^{j+1} d_{2(p-j)}} \quad(j=\overline{0, p-1}), \\
\alpha_{p p}=\frac{B_{p}}{3 \cdot 4^{p+1}}, \quad \beta_{p p}=\frac{C_{p}}{3 \cdot 4^{p+1}} .
\end{gathered}
$$

Now we formulate a result from [4] in a suitable for us form. 
Theorem 1.1. Let $p \in N, x \in \widetilde{C}^{p}([0, \omega]), x^{(i)}(0)=x^{(i)}(\omega)(i=0, \ldots, p)$, and let $d_{p}$ be given by the equalities (1.4) and (1.5). Assume, moreover, that $x$ is not equal identically to a constant. Then

$$
\Delta(x)<\frac{\omega^{p}}{d_{p}} \Delta\left(x^{(p)}\right) .
$$

Remark 1.1. It was shown in [4] that

$$
d_{4}=\frac{2^{11} \cdot 3}{5}, \quad d_{5}=2^{9} \cdot 3 \cdot 5, \quad d_{6}=\frac{2^{16} \cdot 3^{2} \cdot 5}{61}, \quad d_{7}=\frac{2^{14} \cdot 3^{2} \cdot 5 \cdot 7}{17},
$$

and the numbers $d_{k}, k=\overline{1,7}$, are optimal in the sense that (1.6) cannot be replaced by the inequality

$$
\Delta(x)<\frac{\omega^{p}}{d_{p}+\varepsilon} \Delta\left(x^{(p)}\right)
$$

no matter how small $\varepsilon \in] 0,1]$ would be.

Definition 1.2. With system (1.1), we associate the matrix $A_{1}=\left(a_{i, j}^{(1)}\right)_{i, j=1}^{n}$ defined by the equalities

$$
\begin{gathered}
a_{1,1}^{(1)}=-1, \quad a_{n, 1}^{(1)}=\frac{\left\|\ell_{n, 1}\right\|}{4}, \quad a_{i, 1}^{(1)}=0 \text { for } 2 \leq i \leq n-1, \\
a_{i+1, i+1}^{(1)}=\left\|\ell_{i+1, i+1}\right\|-\frac{d_{p_{i+1}-1}}{\omega^{p_{i+1}-1}}, \quad a_{i, i+1}^{(1)}=\frac{\left\|\ell_{n, 1}\right\|}{4} \text { for } 1 \leq i \leq n-1, \\
a_{i, j}^{(1)}=0 \text { for } i+2 \leq j \leq n, \quad a_{i, j}^{(1)}=\left\|\ell_{i, j}\right\| \text { for } 3 \leq j+1 \leq i \leq n,
\end{gathered}
$$

and the matrices $A_{k}=\left(a_{i, j}^{(k)}\right)_{i, j=1}^{n}(k=\overline{2, n})$ given by the recurrence relations

$$
\begin{gathered}
A_{2}=A_{1}, \\
a_{i, j}^{(k+1)}=a_{i, j}^{(k)} \quad \text { for } i \leq k \text { or } j \notin\{k, k+1\}, \\
a_{i, j}^{(k+1)}=a_{i, j}^{(k)}+\frac{a_{k, j}^{(k)}}{\left|a_{k, k}^{(k)}\right|} a_{i, k}^{(k)} \quad \text { for } k+1 \leq i \leq n, \quad k \leq j \leq k+1 .
\end{gathered}
$$

Theorem 1.2. Let $\ell_{n, 1}, \ell_{i, i+1}: C([0, \omega]) \rightarrow L([0, \omega]) \quad(i=\overline{1, n-1})$ be linear monotone operators,

$$
\int_{0}^{\omega} \ell_{n, 1}(1)(s) d s \neq 0, \quad \int_{0}^{\omega} \ell_{i, i+1}(1)(s) d s \neq 0 \quad \text { for } \quad 1 \leq i \leq n-1,
$$
and

$$
a_{k, k}^{(k)}<0 \quad \text { for } \quad 2 \leq k \leq n
$$


where the matrices $A_{k}$ are defined by relations (1.7)-(1.10). Let, moreover,

$$
\int_{0}^{\omega}\left|\ell_{n, 1}(1)(s)\right| d s \prod_{j=1}^{n-1} \int_{0}^{\omega}\left|\ell_{j, j+1}(1)(s)\right| d s<4^{n} \prod_{j=2}^{n}\left|a_{j, j}^{(j)}\right| .
$$

Then problem (1.1), (1.2) has a unique solution.

Definition 1.3. For the system (1.3) we define the matrix $A_{1}=\left(a_{i, j}^{(1)}\right)_{i, j=1}^{n}$ by the equalities (1.7)-(1.10) with

$$
\ell_{i, j}(x)(t)=h_{i, j}(t) x(t) \quad \text { for } \quad i, j \in \overline{1, n}, \quad x \in C([0, \omega]) .
$$

Corollary 1.1. Let

$$
0 \leq \sigma_{n} h_{n, 1}(t) \not \equiv 0,0 \leq \sigma_{i} h_{i, i+1}(t) \not \equiv 0 \quad \text { for } \quad 1 \leq i \leq n-1
$$

where $\sigma_{i} \in\{-1,1\}(i=\overline{1, n})$, the matrices $A_{k}$ are defined by the relations (1.8)(1.10), (1.14) and

$$
a_{k, k}^{(k)}<0 \quad \text { for } \quad 2 \leq k \leq n .
$$

Let, moreover,

$$
\int_{0}^{\omega}\left|h_{n, 1}(s)\right| d s \prod_{j=1}^{n-1} \int_{0}^{\omega}\left|h_{j, j+1}(s)\right| d s<4^{n} \prod_{j=2}^{n}\left|a_{j, j}^{(j)}\right| .
$$

Then problem (1.3), (1.2) has a unique solution.

Now, assume that

$$
\begin{gathered}
\ell_{1, j} \equiv 0 \text { for } j \neq 2, \quad \ell_{i, j} \equiv 0 \text { for } j \notin\{i, i+1\}, i=\overline{2, n-1}, \\
\ell_{n, j}=0 \text { for } j=\overline{2, n-1} .
\end{gathered}
$$

Then system (1.1) is of the following type:

$$
\begin{aligned}
& u_{1}^{\left(p_{1}\right)}(t)=\ell_{1,2}\left(u_{2}\right)(t)+q_{1}(t), \\
& u_{i}^{\left(p_{i}\right)}(t)=\ell_{i, i}\left(u_{i}\right)(t)+\ell_{i, i+1}\left(u_{i+1}\right)(t)+q_{i}(t) \text { for } 2 \leq i \leq n-1, \\
& u_{n}^{\left(p_{n}\right)}(t)=\ell_{n, 1}\left(u_{1}\right)(t)+\ell_{n, n}\left(u_{n}\right)(t)+q_{n}(t),
\end{aligned}
$$

and from Theorem 1.2 we obtain

Corollary 1.2. Let $\ell_{n, 1}, \ell_{i, i+1}(i=\overline{1, n-1})$ be linear monotone operators, the conditions (1.11) hold and, moreover,

$$
\int_{0}^{\omega}\left|\ell_{k, k}(1)(s)\right| d s<\frac{d_{p_{k}-1}}{\omega^{p_{k}-1}} \quad \text { for } \quad 2 \leq k \leq n .
$$


Let, moreover,

$$
\begin{aligned}
\int_{0}^{\omega}\left|\ell_{n, 1}(1)(s)\right| d s \prod_{j=1}^{n-1} \int_{0}^{\omega}\left|\ell_{j, j+1}(1)(s)\right| d s \\
<4^{n} \prod_{j=2}^{n}\left(\frac{d_{p_{j}-1}}{\omega^{p_{j}-1}}-\int_{0}^{\omega}\left|\ell_{j, j}(1)(s)\right| d s\right) .
\end{aligned}
$$

Then problem (1.19), (1.2) has a unique solution.

For the cyclic feedback system

$$
\begin{aligned}
& u_{i}^{\left(p_{1}\right)}(t)=\ell_{i}\left(u_{i+1}\right)(t)+q_{i}(t) \quad \text { for } \quad 1 \leq i \leq n-1, \\
& u_{n}^{\left(p_{n}\right)}(t)=\ell_{n}\left(u_{1}\right)(t)+q_{n}(t),
\end{aligned}
$$

Corollary 1.2 yields

Corollary 1.3. Let $\ell_{i}: C([0, \omega]) \rightarrow L([0, \omega])(i=\overline{1, n})$ be linear monotone operators,

$$
\left\|\ell_{i}\right\| \neq 0 \quad \text { for } \quad i=\overline{1, n}
$$

and

$$
\prod_{i=1}^{n}\left\|\ell_{i}\right\|<4^{n} \prod_{j=2}^{n} \frac{d_{p_{j}-1}}{\omega^{p_{j}-1}} .
$$

Then problem (1.22), (1.2) has a unique solution.

Remark 1.2. The problem

$$
u^{\prime \prime}(t)=p(t) u(t)+q(t), u(0)=u(\omega), u^{\prime}(0)=u^{\prime}(\omega),
$$

is equivalent to the problem (1.22), (1.2) with $n=2, n_{1}=n_{2}=1 \ell_{1}(x)(t)=$ $x(t), \ell_{2}(x)(t)=p(t) x(t), q_{1} \equiv 0$ and $q_{2} \equiv q$.

Then if $p, q \in L([0, \omega]), p(t) \leq 0$, and $\int_{0}^{\omega} p(s) d s \neq 0$, it follows from Corollary 1.3 that problem (1.22), (1.2) and, therefore, problem (1.25), has a unique solution provided that the condition

$$
\int_{0}^{\omega}|p(s)| d s<\frac{16}{\omega}
$$

is fulfilled. This follows from a well-known result of A. Lasota and Z. Opial (see [7]).

Remark 1.3. Rewrite the problem

$$
u^{(4)}(t)=\ell(u)(t)+q(t), \quad u^{(i)}(0)=u^{(i)}(\omega) \quad(i=0,1,2,3)
$$


as the systems

$$
\begin{gathered}
u_{i}^{\prime}(t)=u_{i+1}(t)(i=1,2,3), \\
u_{4}^{\prime}(t)=\ell\left(u_{1}\right)(t)+q(t), \\
u_{i}(0)=u_{i}(\omega)(i=1,2,3,4)
\end{gathered}
$$

and

$$
\begin{gathered}
u_{1}^{\prime}(t)=u_{2}(t), \\
u_{2}^{(3)}(t)=\ell\left(u_{1}\right)(t)+q(t), \\
u_{1}(0)=u_{1}(\omega), u_{2}^{(j)}(0)=u_{2}^{(j)}(\omega) \quad(j=0,1,2) .
\end{gathered}
$$

It follows from [12, Theorem 3] that problem (1.26), i.e., problems (1.27) and (1.28), are uniquely solvable if $\|\ell\| \leq \frac{768}{\omega^{3}}$, and the constant 768 is optimal.

On the other hand, it follows from Corollary 1.3 that system (1.27) (resp., (1.28)) is uniquely solvable if $\|\ell\| \leq \frac{256}{\omega^{3}}$ (resp., $\|\ell\| \leq \frac{512}{\omega^{3}}$ ). Consequently, if we rewrite every equation of (1.1) as a system of first order equations, we debase the quality of uniqueness condition.

Example 1.1. The example below shows that condition (1.24) in Corollary 1.3 is optimal and cannot be replaced by the condition

$$
\prod_{i=1}^{n}\left\|\ell_{i}\right\| \leq 4^{n} \prod_{j=2}^{n} \frac{d_{p_{j}-1}}{\omega^{p_{j}-1}} .
$$

Define the function $u_{0} \in \widetilde{C}([0,1])$ on $[0,1 / 2]$, and extend it to $[1 / 2,1]$ by the equalities

$$
u_{0}(t)= \begin{cases}1 & \text { for } 0 \leq t \leq 1 / 8 \\ \sin \pi(1-4 t) & \text { for } 1 / 8<t \leq 3 / 8 \\ -1 & \text { for } 3 / 8<t \leq 1 / 2\end{cases}
$$

and

$$
u_{0}(t)=u_{0}(1-t) \text { for } 1 / 2<t \leq 1 .
$$

Now let measurable functions $\tau_{i}:[0,1] \rightarrow[0,1]$ and the linear nonnegative operators $\ell_{i}: C([0,1]) \rightarrow L([0,1])(i=\overline{1, n})$ be given by the equalities

$$
\tau_{i}(t)=\left\{\begin{array}{ll}
1 / 8 i & \text { for } 0 \leq u_{0}^{\prime}(t) \\
1 / 2-1 / 8 i & \text { for } 0>u_{0}^{\prime}(t)
\end{array}, \quad \ell_{i}(x)(t)=\left|u_{0}^{\prime}(t)\right| x\left(\tau_{i}(t)\right) .\right.
$$

Then it is clear that $u_{0}(0)=u_{0}(1), \ell_{i} \neq \ell_{j}$ if $i \neq j$, and $\left\|\ell_{i}\right\|=\int_{0}^{1}\left|\ell_{i}(1)(s)\right| d s=$ $16 \pi \int_{1 / 8}^{1 / 4} \cos \pi(1-4 s) d s=4$ for $i=\overline{1, n}$. Thus, all the assumptions of Corollary 1.3 are satisfied except (1.24), instead of which condition (1.24 $)$ is fulfilled with $\omega=1$. On the other hand, from the relations $u_{0}^{\prime}(t)=\left|u_{0}^{\prime}(t)\right| u_{0}\left(\tau_{i}(t)\right)=\ell_{i}\left(u_{0}\right)(t) \quad(i=$ $\overline{1, n})$, it follows that the vector function $\left(u_{i}(t)\right)_{i=1}^{n}$ if $u_{i}(t) \equiv u_{0}(t) \quad(i=\overline{1, n})$, is a 
nontrivial solution of problem (1.22), (1.2) with $\omega=1, q(t) \equiv 0, p_{j}=1(j=\overline{1, n})$, which contradicts the conclusion of Corollary 1.3.

\section{AUXILIARY PROPOSITIONS}

Lemma 2.1. Let the matrices $A_{k}(k=\overline{1, n})$ be defined by equalities (1.7)-(1.10). Then the following relations hold:

$$
\begin{aligned}
& a_{i, j}^{(m)} \geq 0 \quad \text { for } \quad i \neq j, \quad m=\overline{1, n}, \\
& a_{n, 1}^{(1)}=a_{n, 1}^{(n)} \\
& a_{i, j}^{(\lambda)} \leq a_{i, j}^{(m)} \quad \text { for } \quad i \geq m \geq 2, j \geq m, \lambda \leq m .
\end{aligned}
$$

Proof. It follows immediately from the definition of $A_{1}$ and $A_{2}$ that inequalities $\left(2.1_{1}\right)$ and $\left(2.2_{2}\right)$ are true. Assume now that $\left(2.1_{m}\right)$ holds for $m=3, \ldots, m_{0}\left(m_{0}<n\right)$ and prove $\left(2.1_{m_{0}+1}\right)$. If $i \leq m_{0}$ or $j \notin\left\{m_{0}, m_{0}+1\right\}$, relation (1.9) implies inequality $\left(2.1_{m_{0}+1}\right)$, and if $i \geq m_{0}+1, j \in\left\{m_{0}, m_{0}+1\right\}$, then (2.1 $\left.1_{m_{0}+1}\right)$ follows from (1.10).

Now we prove inequality $\left(2.2_{m}\right)$. First assume that $j \geq m+1$. Then from (1.9) with $k=m-1$ it is clear that

$$
a_{i, j}^{(\lambda)}=a_{i, j}^{(\lambda+1)}=\cdots=a_{i, j}^{(m)} \quad \text { for } \quad j \geq m+1, i \geq m, \lambda \leq m .
$$

Now, let $j=m$. Then from (1.9) we get $a_{i, m}^{(\lambda)}=a_{i, m}^{(\lambda+1)}=\cdots=a_{i, m}^{(m-1)}$ for $i \geq m, \lambda \leq$ $m-1$. By the last equalities and $\left(2.1_{m}\right)$, from (1.10) it follows that

$$
a_{i, m}^{(m)}=a_{i, m}^{(m-1)}+\frac{a_{m-1, m}^{(m-1)}}{\left|a_{m-1, m-1}^{(m-1)}\right|} a_{i, m-1}^{(m-1)} \geq a_{i, m}^{(m-1)}=a_{i, m}^{(\lambda)} \quad \text { for } \quad i \geq m, \lambda \leq m .
$$

From this inequality and (2.3) we conclude that $(2.2 \mathrm{~m})$ is fulfilled for all $j \geq m$ and $i \geq m$. Equality (2.20) follows immediately from (1.8) and (1.9).

Also we need the following simple lemma proved in the paper [11].

Lemma 2.2. Let $\sigma \in\{-1,1\}$ and $\sigma \ell: C([0, \omega]) \rightarrow L([0, \omega])$ be a nonnegative linear operator. Then

$$
-m|\ell(1)(t)| \leq \sigma \ell(x)(t) \leq M|\ell(1)(t)| \quad \text { for } \quad 0 \leq t \leq \omega, \quad x \in C([0, \omega]),
$$

where $m=-\min _{0 \leq t \leq \omega}\{x(t)\}$ and $M=\max _{0 \leq t \leq \omega}\{x(t)\}$.

Now, consider on $[0, \omega]$ the homogeneous problem

$$
\begin{gathered}
v_{i}^{\left(p_{i}\right)}(t)=\sum_{j=2}^{i+1} \ell_{i, j}\left(v_{j}\right)(t) \quad \text { for } \quad 1 \leq i \leq n, \\
v_{j}^{(p)}(0)=v_{j}^{(p)}(\omega) \quad \text { for } \quad 1 \leq j \leq n, 0 \leq p \leq p_{j}-1,
\end{gathered}
$$

where the operator $\ell_{n, n+1}$ and function $v_{n+1}$ are defined by the equalities $\ell_{n, n+1} \equiv$ $\ell_{n, 1}$ and $v_{n+1} \equiv v_{1}$. Then it is clear that $\Delta\left(v_{n+1}\right) \equiv \Delta\left(v_{1}\right)$. 
Lemma 2.3. Let $\ell_{i, i+1}: C([0, \omega]) \rightarrow L([0, \omega])(i=\overline{1, n})$ be linear monotone operators,

$$
\int_{0}^{\omega} \ell_{i, i+1}(1)(s) d s \neq 0 \quad \text { for } \quad 1 \leq i \leq n,
$$

the matrices $A_{k}$ be defined by the equalities (1.7)-(1.10) and

$$
a_{k, k}^{(k)}<0 \quad \text { for } \quad 2 \leq k \leq n .
$$

Let, moreover $v=\left(v_{i}\right)_{i=1}^{n}$ be a nontrivial solution of the problem $\left(\left(2.4_{i}\right)\right)_{i=1}^{n}$, (2.5) for which there exists a $k_{1} \in\{2, \ldots, n\}$ such that $v_{k_{1}} \not \equiv 0$. Then if

$$
k_{0}=\min \left\{k \in\{2, \ldots, n\}: v_{k} \not \equiv 0\right\}
$$

the inequalities

$$
\begin{array}{r}
0<\left\|v_{k}\right\|_{C} \leq \Delta\left(v_{k}\right) \quad \text { for } \quad k=1, k_{0} \leq k \leq n, \\
0 \leq \frac{\omega^{p_{k}-1}}{d_{p_{k}-1}} a_{k, k}^{(k)} \Delta\left(v_{k}^{\left(p_{k}-1\right)}\right)+\frac{\omega^{p_{k+1}-1}}{d_{p_{k+1}-1}} a_{k, k+1}^{(k)} \Delta\left(v_{k+1}^{\left(p_{k+1}-1\right)}\right)
\end{array}
$$

for $k_{0} \leq k \leq n$ hold, where $a_{n, n+1}^{(1)}=a_{n, 1}^{(1)}$.

Proof. Define the numbers $M_{k, i}, m_{k, i} \in \mathbb{R}, t_{k, i}^{\prime}, t_{k, i}^{\prime \prime} \in[0, \omega]\left(k=\overline{1, n}, i=1, p_{k}-\right.$ 1) by the relations

$$
\begin{aligned}
& M_{k, i}=v_{k}^{(i)}\left(t_{k, i}^{\prime}\right)=\max _{0 \leq t \leq \omega}\left\{v_{k}^{(i)}(t)\right\}, \\
& -m_{k, i}=v_{k}^{(i)}\left(t_{k, i}^{\prime \prime}\right)=\min _{0 \leq t \leq \omega}\left\{v_{k}^{(i)}(t)\right\},
\end{aligned}
$$

and introduce the sets $I_{k}^{(1)}=\left[t_{k, p_{k}-1}^{\prime}, t_{k, p_{k}-1}^{\prime \prime}\right], I_{k}^{(2)}=I \backslash I_{k}^{(1)}$ for $t_{k, p_{k}-1}^{\prime}<t_{k, p_{k}-1}^{\prime \prime}$. It is clear from (2.8) that

$$
v_{k_{0}} \not \equiv 0
$$

On the other hand, from $\left(2.4_{k_{0}-1}\right)$ by $(2.8)$ we obtain

$$
\int_{0}^{\omega} \ell_{k_{0}-1, k_{0}}\left(v_{k_{0}}\right)(s) d s=0
$$

Equality (2.13), in view of (2.6) and Lemma 2.2 guarantees the existence of a $t_{0} \in$ $[0, \omega]$ such that $v_{k_{0}}\left(t_{0}\right)=0$. Then from (2.12) we get $\left(2.9_{k_{0}}\right)$.

Let the numbers $M_{k_{0}, p_{k_{0}}-1}, m_{k_{0}, p_{k_{0}}-1}, M_{k_{0}+1,1}, m_{k_{0}+1,1} \in \mathbb{R}, t_{k_{0}}^{\prime}, t_{k_{0}}^{\prime \prime} \in[0, \omega]$ be defined by the relations $\left(2.11_{k}\right)$ and $t_{k_{0}, p_{k_{0}}-1}^{\prime}<t_{k_{0}, p_{k_{0}}-1}^{\prime \prime}$ (the case $t_{k_{0}, p_{k_{0}}-1}^{\prime \prime}<$ $t_{k_{0}, p_{k_{0}}-1}^{\prime}$ can be considered analogously). The integration of $\left(2.4_{k_{0}}\right)$ on $I_{k_{0}}^{(r)}$, by 
virtue of (2.5) and (2.8) results in

$$
\begin{aligned}
\Delta\left(v_{k_{0}}^{\left(p_{k_{0}}-1\right)}\right)=(-1)^{r}\left(\int_{I_{k_{0}}^{(r)}} \ell_{k_{0}, k_{0}}\left(v_{k_{0}}\right)(s) d s\right. & \\
& \left.+\int_{I_{k_{0}}^{(r)}} \ell_{k_{0}, k_{0}+1}\left(v_{k_{0}+1}\right)(s) d s\right)
\end{aligned}
$$

for $r=1,2$. From the last equality, by virtue of (1.7), (2.7), $\left(2.9_{k_{0}}\right)$ and $\left(2.2_{k_{0}}\right)$ with $\lambda=1, i=j=k_{0}$ we get

$$
0<-\frac{\omega^{p_{k_{0}}-1}}{d_{p_{k_{0}}-1}} a_{k_{0}, k_{0}}^{\left(k_{0}\right)} \Delta\left(v_{k_{0}}^{\left(p_{k_{0}}-1\right)}\right) \leq(-1)^{r} \int_{I_{k_{0}}^{(r)}} \ell_{k_{0}, k_{0}+1}\left(v_{k_{0}+1}\right)(s) d s
$$

for $r=1,2$. Assume that $v_{k_{0}+1}$ is a constant sign function. Then, in view of the fact that the operator $\ell_{k_{0}, k_{0}+1}$ is monotone, we get a contradiction with (2.151) or $\left(2.15_{2}\right)$, i. e., $v_{k_{0}+1}$ changes its sign. Then

$$
M_{k_{0}+1,1}>0, m_{k_{0}+1,1}>0,
$$

and the inequality $\left(2.9_{k_{0}+1}\right)$ holds $\left(\left(2.9_{1}\right)\right.$ if $\left.k_{0}=n\right)$. If $\ell_{k_{0}, k_{0}+1}$ is a non-negative operator, from $\left(2.15_{r}\right)(r=1,2)$ in view of $(2.16)$ by Lemma 2.2 , we get

$$
\begin{aligned}
& 0<-\frac{\omega^{p_{k_{0}}-1}}{d_{p_{k_{0}}-1}} a_{k_{0}, k_{0}}^{\left(k_{0}\right)} \Delta\left(v_{k_{0}}^{\left(p_{k_{0}}-1\right)}\right) \leq m_{k_{0}+1} \int_{I_{k_{0}}^{(1)}}\left|\ell_{k_{0}, k_{0}+1}(1)(s)\right| d s, \\
& 0<-\frac{\omega^{p_{k_{0}}-1}}{d_{p_{k_{0}}-1}} a_{k_{0}, k_{0}}^{\left(k_{0}\right)} \Delta\left(v_{k_{0}}^{\left(p_{k_{0}}-1\right)}\right) \leq M_{k_{0}+1} \int_{I_{k_{0}}^{(2)}}\left|\ell_{k_{0}, k_{0}+1}(1)(s)\right| d s .
\end{aligned}
$$

By multiplying these estimates and applying the numerical inequality $4 A B \leq(A+$ $B)^{2}$, in view of (1.6) and notation (1.7), we obtain

$$
\begin{aligned}
0 & \leq \frac{\omega^{p_{k_{0}}-1}}{d_{p_{k_{0}}-1}} a_{k_{0}, k_{0}}^{\left(k_{0}\right)} \Delta\left(v_{k_{0}}^{\left(p_{k_{0}}-1\right)}\right) \\
& +\frac{1}{4}\left(M_{k_{0}+1,1}+m_{k_{0}+1,1}\right)\left(\int_{I_{k_{0}}^{(1)}}\left|\ell_{k_{0}, k_{0}+1}(1)(s)\right| d s+\int_{I_{k_{0}}^{(2)}}\left|\ell_{k_{0}, k_{0}+1}(1)(s)\right| d s\right) \\
& \leq \frac{\omega^{p_{k_{0}}-1}}{d_{p_{k_{0}}-1}} a_{k_{0}, k_{0}}^{\left(k_{0}\right)} \Delta\left(v_{k_{0}}^{\left(p_{k_{0}}-1\right)}\right)+\frac{\omega^{p_{k_{0}+1}-1}}{d_{p_{k_{0}+1}-1}} a_{k_{0}, k_{0}+1}^{(1)} \Delta\left(v_{k_{0}+1}^{\left(p_{k_{0}+1}-1\right)}\right),
\end{aligned}
$$

$\left(0 \leq \frac{\omega^{p_{n}-1}}{d_{p_{n}-1}} a_{n, n}^{(n)} \Delta\left(v_{n}^{\left(p_{n}-1\right)}\right)+\frac{\omega^{p_{1}-1}}{d_{p_{1}-1}} a_{n, 1}^{(1)} \Delta\left(v_{1}^{\left(p_{1}-1\right)}\right)\right.$ if $\left.k_{0}=n\right)$, whence, by virtue of $\left(2.2_{0}\right)$ if $k_{0}=n$ and $\left(2.2_{k_{0}}\right)$ with $\lambda=1, i=k_{0}, j=k_{0}+1$ if $k_{0}<n$, the relation $\left(2.10_{k_{0}}\right)$ follows. Analogously, from $\left(2.15_{r}\right)$ we get $\left(2.10_{k_{0}}\right)$ in the case where the operator $\ell_{k_{0}, k_{0}+1}$ is nonpositive.

We have thus proved the proposition: 
(i) Let $2 \leq k_{0} \leq n$, then the inequalities $\left(2.9_{k_{0}}\right),\left(2.9_{k_{0}+1}\right)\left(\left(2.9_{1}\right)\right.$ if $\left.k_{0}=n\right)$ and $\left(2.10_{k_{0}}\right)$ hold.

Now, we shall prove the following proposition:

(ii) Let $k_{1} \in\left\{k_{0}, \ldots, n-1\right\}$ be such that the inequalities $\left(2.9_{k}\right),\left(2.10_{k}\right)$ for $(k=$ $\left.\overline{k_{0}, k_{1}}\right)$, and $\left(2.9_{k_{1}+1}\right)$ hold. Then the inequalities $\left(2.9_{k_{1}+2}\right)$ if $k_{1} \leq n-2$, $\left(2.9_{1}\right)$ if $k_{1}=n-1$ and $\left(2.10_{k_{1}+1}\right)$ hold too.

Define the numbers

$M_{k_{1}+1, p_{k_{1}+1}-1}, m_{k_{1}+1, p_{k_{1}+1}-1} \in \mathbb{R}$ and $t_{k_{1}+1, p_{k_{1}+1}-1}^{\prime}, t_{k_{1}+1, p_{k_{1}+1}-1}^{\prime \prime} \in[0, \omega]$,

by the relations $\left(2.11_{k_{1}+1}\right)$ and let $t_{k_{1}+1, p_{k_{1}+1}-1}^{\prime}<t_{k_{1}+1, p_{k_{1}+1}-1}^{\prime \prime}$ (the case where $t_{k_{1}+1, p_{k_{1}+1}-1}^{\prime \prime}<t_{k_{1}+1, p_{k_{1}+1}-1}^{\prime}$ can be considered analogously). The integration of $\left(2.4_{k_{1}+1}\right)$ on $I_{k_{1}+1}^{(r)}$, by virtue of (2.5) and (2.8) results in

$$
\Delta\left(v_{k_{1}+1}^{\left(p_{k_{1}+1}-1\right)}\right)=(-1)^{r} \sum_{j=k_{0}}^{k_{1}+2} \int_{I_{k_{1}+1}^{(r)}} \ell_{k_{1}+1, j}\left(v_{j}\right)(s) d s
$$

for $r=1,2$. From this equality, by the conditions (1.6), (1.7), (2.7), (2.9k) with $k=k_{0}, \ldots, k_{1}+1$, and $\left(2.2_{k_{0}}\right)$ with $\lambda=1, i=k_{1}+1, j=k_{0}, \ldots, k_{1}+1$ we get

$$
\begin{aligned}
0 \leq \sum_{j=k_{0}}^{k_{1}+1} \frac{\omega^{p_{j}-1}}{d_{p_{j}-1}} a_{k_{1}+1, j}^{\left(k_{0}\right)} \Delta\left(v_{j}^{\left(p_{j}-1\right)}\right) & \\
& +(-1)^{r} \int_{I_{k_{1}+1}^{(r)}} \ell_{k_{1}+1, k_{1}+2}\left(v_{k_{1}+2}\right)(s) d s
\end{aligned}
$$

for $r=1$, 2. By multiplying $\left(2.10_{k}\right)$ with $a_{k_{1}+1, k}^{(k)} /\left|a_{k, k}^{(k)}\right|$ for $k \in\left\{k_{0}, \ldots, k_{1}\right\}$ in view of the inequalities (2.7) we obtain

$$
\begin{aligned}
0 \leq-\frac{\omega^{p_{k}-1}}{d_{p_{k}-1}} a_{k_{1}+1, k}^{(k)} \Delta\left(v_{k}^{\left(p_{k}-1\right)}\right) & \\
& +\frac{\omega^{p_{k+1}-1}}{d_{p_{k+1}-1}} \frac{a_{k, k+1}^{(k)}}{\left|a_{k, k}^{(k)}\right|} a_{k_{1}+1, k}^{(k)} \Delta\left(v_{k+1}^{\left(p_{k+1}-1\right)}\right) .
\end{aligned}
$$


Now, summing (2.18) and $\left(2.19_{k_{0}}\right)$, by virtue of (1.10) with $k=k_{0}, i=k_{1}+1, j=$ $k_{0}+1$, we get the estimate

$$
\begin{array}{r}
0 \leq \frac{\omega^{p_{k_{0}+1}-1}}{d_{p_{k_{0}+1}-1}} a_{k_{1}+1, k_{0}+1}^{\left(k_{0}+1\right)} \Delta\left(v_{k_{0}+1}^{\left(p_{k_{0}+1}-1\right)}\right) \\
+\sum_{j=k_{0}+2}^{k_{1}+1} \frac{\omega^{p_{j}-1}}{d_{p_{j}-1}} a_{k_{1}+1, j}^{\left(k_{0}\right)} \Delta\left(v_{j}^{\left(p_{j}-1\right)}\right) \\
+(-1)^{r} \int_{I_{k_{1}+1}^{(r)}} \ell_{k_{1}+1, k_{1}+2}\left(v_{k_{1}+2}\right)(s) d s,
\end{array}
$$

from which, by $\left(2.2_{k_{0}+1}\right)$ with $i=k_{1}+1, j \geq k_{0}+2, \lambda=k_{0}$, we obtain

$$
\begin{aligned}
0 \leq \sum_{j=k_{0}+1}^{k_{1}+1} \frac{\omega^{p_{j}-1}}{d_{p_{j}-1}} a_{k_{1}+1, j}^{\left(k_{0}+1\right)} \Delta\left(v_{j}^{\left(p_{j}-1\right)}\right) & \\
& +(-1)^{r} \int_{I_{k_{1}+1}^{(r)}} \ell_{k_{1}+1, k_{1}+2}\left(v_{k_{1}+2}\right)(s) d s
\end{aligned}
$$

for $r=1,2$. Analogously, by summing (2.20) and the inequalities $\left(2.19_{k}\right)$ for all $k=k_{0}+1, \ldots, k_{1}$ we get

$$
\begin{aligned}
0<-\frac{\omega^{p_{k_{1}+1}-1}}{d_{p_{k_{1}+1}-1}} a_{k_{1}+1, k_{1}+1}^{\left(k_{1}+1\right)} \Delta( & \left.v_{k_{1}+1}^{\left(p_{k_{1}+1}-1\right)}\right) \\
& \leq(-1)^{r} \int_{I_{k_{1}+1}^{(r)}} \ell_{k_{1}+1, k_{1}+2}\left(v_{k_{1}+2}\right)(s) d s
\end{aligned}
$$

for $r=1$, 2. In the same way as the inequality $\left(2.9_{k_{0}+1}\right)$ and $\left(2.10_{k_{0}}\right)$ follow from $\left(2.15_{r}\right)$, the inequalities $\left(2.9_{k_{1}+2}\right)\left(\left(2.9_{1}\right)\right.$ if $\left.k_{0}=n-1\right)$ and $\left(2.10_{k_{1}+1}\right)$ follow from (2.21).

From the propositions (i) and (ii), by the method of mathematical induction, we obtain that the inequalities $\left(2.9_{1}\right),\left(2.9_{k}\right)$ and $\left(2.10_{k}\right)\left(k=\overline{k_{0}, n}\right)$ hold.

\section{PROOFS}

Proof of Theorem 1.2. It is known from the general theory of boundary value problems for functional differential equations that if $\ell_{i, j}(i, j=\overline{1, n})$ are bounded linear operators, then problem (1.1), (1.2) has the Fredholm property (see [3]). Thus, problem (1.1), (1.2) is uniquely solvable if and only if the homogeneous problem $\left(2.4_{i}\right)_{i=1}^{n},(2.5)$ has only the trivial solution.

Assume that, on the contrary, the problem $\left(2.4_{i}\right)_{i=1}^{n},(2.5)$ has a nontrivial solution $v=\left(v_{i}\right)_{i=1}^{n}$. Let

$$
v_{1} \not \equiv 0, \quad v_{i} \equiv 0 \quad \text { for } \quad 2 \leq i \leq n .
$$

Thus, from $\left(2.4_{1}\right)$ and $\left(2.4_{n}\right)$, it follows that

$$
v_{1}^{\left(p_{1}\right)} \equiv 0,
$$


and $\ell_{n, 1}\left(v_{1}\right)(t) \equiv 0$. Then from (1.11) we obtain that $v_{1} \not \equiv$ const, whence, due to conditions (2.5), it follows $v_{1}^{\prime} \not \equiv$ const. Also it is clear that from the conditions $v_{1}^{(i)} \not \equiv$ const and (2.5) it follows $v_{1}^{(i+1)} \not \equiv$ const. Thus, by the method of mathematical induction, we obtain that $v_{1}^{\left(p_{1}\right)} \not \equiv$ const, which contradicts (3.2) and, therefore, contradicts (3.1). Consequently, there exists $k_{0} \in\{2, \ldots, n\}$ such that $v_{k_{0}} \not \equiv 0$. Then all the conditions of Lemma 2.3 are satisfied, from which we conclude that $0<\left\|v_{1}\right\|_{C} \leq \Delta\left(v_{1}\right)$, i. e., $v_{1} \not \equiv$ const and in view of the condition (2.5) the function $v_{1}^{\left(p_{1}\right)}$ changes its sign. Thus, from $\left(2.4_{1}\right)$, by the monotonicity of the operator $\ell_{1,2}$, we get that $v_{2}$ also changes its sign. Consequently, if $M_{2,1}$ and $m_{2,1}$ are the numbers defined by the equalities $\left(2.11_{2}\right)$, then

$$
M_{2,1}>0, \quad m_{2,1}>0,
$$

and if $k_{0}$ is the number defined by the equality (2.8), then $k_{0}=2$. Thus, it follows from Lemma 2.3 that the inequalities $\left(2.9_{1}\right),\left(2.9_{k}\right)$ and $\left(2.10_{k}\right)(k=\overline{2, n})$ hold.

Now, assume that the numbers $M_{1, p_{1}-1}, m_{1, p_{1}-1}$, and $t_{1, p_{1}-1}^{\prime}, t_{1, p_{1}-1}^{\prime \prime} \in[0, \omega[$ are defined by the equalities $\left(2.11_{1}\right)$ and $t_{1, p_{1}-1}^{\prime}<t_{1, p_{1}-1}^{\prime \prime}$ (the case where $t_{1, p_{1}-1}^{\prime \prime}<$ $t_{1, p_{1}-1}^{\prime}$ is considered analogously). By integration of $\left(2.4_{1}\right)$ on the set $I_{1}^{(r)}$ and by using the inequality $\left(2.9_{1}\right)$, we obtain

$$
0<\Delta\left(v_{1}^{\left(p_{1}-1\right)}\right)=(-1)^{r} \int_{I_{1}^{(r)}} \ell_{1,2}\left(v_{2}\right)(s) d s
$$

for $r=1,2$. Let us first assume that the operator $\ell_{1,2}$ is nonnegative (the case of a nonpositive $\ell_{1,2}$ can be considered analogously), then from (3.4) by (3.3) and Lemma 2.2 we obtain

$$
\begin{aligned}
& 0<\Delta\left(v_{1}^{\left(p_{1}-1\right)}\right) \leq m_{2} \int_{I_{1}^{(1)}}\left|\ell_{1,2}(1)(s)\right| d s, \\
& 0<\Delta\left(v_{1}^{\left(p_{1}-1\right)}\right) \leq M_{2} \int_{I_{1}^{(2)}}\left|\ell_{1,2}(1)(s)\right| d s .
\end{aligned}
$$

By multiplying these estimates and applying the numerical equality $4 A B \leq(A+B)^{2}$ and the equalities (1.7), we get

$$
\begin{aligned}
0 & \leq a_{1,1}^{(1)} \Delta\left(v_{1}^{\left(p_{1}-1\right)}\right)+\frac{1}{4}\left(m_{2}+M_{2}\right)\left(\int_{I_{1}^{(1)}}\left|\ell_{1,2}(1)(s)\right| d s+\int_{I_{1}^{(2)}}\left|\ell_{1,2}(1)(s)\right| d s\right) \\
& =a_{1,1}^{(1)} \Delta\left(v_{1}^{\left(p_{1}-1\right)}\right)+a_{1,2}^{(1)} \Delta\left(v_{2}^{\left(p_{2}-1\right)}\right),
\end{aligned}
$$

i. e., all the inequalities $\left(2.10_{k}\right)(k=\overline{1, n})$ are satisfied.

On the other hand from (1.7)-(1.9) and Lemma 2.1 it is clear that

$$
a_{1,1}^{(1)}=-1, \quad a_{n, 1}^{(n)}=a_{n, 1}^{(1)}, \quad a_{k, k+1}^{(k)}=a_{k, k+1}^{(1)}=\frac{1}{4}\left\|\ell_{k, k+1}\right\|
$$


for $1 \leq k \leq n-1$. By multiplying all the estimates $\left(2.10_{k}\right)(k=\overline{1, n})$ and applying (3.5), we get the contradiction with condition (1.13). Thus, our assumption fails, and hence $v_{i} \equiv 0(i=\overline{1, n})$.

Proof of Corollary 1.1. From (1.14) and (1.15) it is clear that $\ell_{n, 1}$ and $\ell_{i, i+1}$ are monotone operators and (1.11) holds. Also, from (1.16) and (1.17), the conditions (1.12) and (1.13) follow. Consequently, all the conditions of Theorem 1.1 are fulfilled for system (1.3).

Proof of Corollary 1.2. From (1.7), (1.9), and (1.18) we obtain

$$
a_{k, k}^{(k-1)}=a_{k, k}^{(k-2)}=\cdots=a_{k, k}^{(1)}=\left\|\ell_{k, k}\right\|-\frac{d_{p_{k}-1}}{\omega^{p_{k}-1}} \quad \text { for } \quad 2 \leq k \leq n,
$$

and

$$
\begin{gathered}
a_{k, k-i}^{(k-i-1)}=a_{k, k-i}^{(k-i-2)}=\cdots=a_{k, k-i}^{(1)}=\left\|\ell_{k, k-i}\right\|=0 \text { for } 3 \leq k-i \leq n, \\
a_{2,1}^{(1)}=0 .
\end{gathered}
$$

From (1.10), (1.18) and the first equality of (3.7) we get

$$
\begin{aligned}
a_{k, k-1}^{(k-1)} & =a_{k, k-1}^{(k-2)}+\frac{a_{k-2, k-1}^{(k-2)}}{\left|a_{k-2, k-2}^{(k-2)}\right|} a_{k, k-2}^{(k-2)}=\frac{a_{k-2, k-1}^{(k-2)}}{\left|a_{k-2, k-2}^{(k-2)}\right|} a_{k, k-2}^{(k-2)} \\
& =\frac{a_{k-2, k-1}^{(k-2)}}{\left|a_{k-2, k-2}^{(k-2)}\right|} \frac{a_{k-3, k-2}^{(k-3)}\left|a_{k-3, k-3}^{(k-3)}\right|}{a_{k, k-3}}=\cdots=a_{k, 2}^{(2)} \prod_{j=2}^{k-2} \frac{a_{j, j+1}^{(j)}}{\left|a_{j, j}^{(j)}\right|}=0
\end{aligned}
$$

for $k \geq 3$. From (3.8) and the second equality of (3.7) it is clear that

$$
a_{k, k-1}^{(k-1)}=0 \quad \text { for } \quad 2 \leq k \leq n .
$$

Then from (1.10) by (3.6) and (3.9) we obtain

$$
a_{k, k}^{(k)}=a_{k, k}^{(k-1)}+a_{k-1, k}^{(k-1)} a_{k, k-1}^{(k-1)} /\left|a_{k-1, k-1}^{(k-1)}\right|=\left\|\ell_{k, k}\right\|-\frac{d_{p_{k}-1}}{\omega^{p_{k}-1}} .
$$

Thus, it follows from the conditions (1.20) and (1.21) that (1.12) and (1.13) hold. Therefore, all the conditions of Theorem 1.1 are fulfilled for the system (1.19).

\section{REFERENCES}

[1] P. W. Bates and J. R. Ward, Jr., "Periodic solutions of higher order systems," Pacific J. Math., vol. 84, no. 2, pp. 275-282, 1979. [Online]. Available: http://projecteuclid.org/getRecord?id= euclid.pjm/1102784209

[2] A. Capietto, D. Qian, and F. Zanolin, "Periodic solutions for differential systems of cyclic feedback type," Differential Equations Dynam. Systems, vol. 7, no. 1, pp. 99-120, 1999.

[3] R. Hakl and S. Mukhigulashvili, "On a boundary value problem for $n$th order linear functional differential systems," Georgian Math. J., vol. 12, no. 2, pp. 229-236, 2005.

[4] R. Hakl and S. Mukhigulashvili, "On one estimate for periodic functions," Georgian Math. J., vol. 12, no. 1, pp. 97-114, 2005. 
[5] I. Kiguradze and S. Mukhigulashvili, "On periodic solutions of two-dimensional nonautonomous differential systems," Nonlinear Anal., vol. 60, no. 2, pp. 241-256, 2005. [Online]. Available: http://dx.doi.org/10.1016/j.na.2004.07.053

[6] I. Kiguradze and B. Půža, "On some boundary value problems for systems of ordinary differential equations," Differents. Uravn., vol. 12, pp. 2139-2148, 1996, in Russian.

[7] A. Lasota and Z. Opial, "Sur les solutions périodiques des équations différentielles ordinaires," Ann. Polon. Math., vol. 16, pp. 69-94, 1964.

[8] A. Lomtatidze and S. Mukhigulashvili, "On periodic solutions of second order functional differential equations," Mem. Differential Equations Math. Phys., vol. 5, pp. 125-126, 1995.

[9] S. Mukhigulashvili, "On periodic solutions of second order functional differential equations," Ital. J. Pure Appl. Math., no. 20, pp. 29-50, 2006.

[10] S. Mukhigulashvili and I. Grytsay, "An optimal condition for the uniqueness of a periodic solution for linear functional differential systems," Electron. J. Qual. Theory Differ. Equ., no. 59, pp. 1-12, 2009.

[11] S. Mukhigulashvili, "On a periodic boundary value problem for cyclic feedback type linear functional differential systems," Arch. Math. (Basel), vol. 87, no. 3, pp. 255-260, 2006. [Online]. Available: http://dx.doi.org/10.1007/s00013-006-1621-1

[12] S. Mukhigulashvili, "On a periodic boundary value problem for fourth order linear functional differential equations," Georgian Math. J., vol. 14, no. 3, pp. 533-542, 2007.

[13] A. Rontó and M. Rontó, "On some symmetric properties of periodic solutions," Nonlinear Oscil. (N. Y.), vol. 6, no. 1, pp. 82-107, 2003.

[14] A. Rontó and M. Rontó, Handbook of Differential Equations: Ordinary Differential Equations. Elsevier, 2008, vol. IV, ch. 5. Successive Approximation Techniques in Non-Linear Boundary Value Problems for Ordinary Differential Equations, pp. 441-592.

Authors' addresses

O. Kuzmych

Volyn National Lesya Ukrainka University, Faculty of Mathematics, 13 Volya Ave., 43000 Lutsk, Ukraine

E-mail address: lenamaks79@mail.ru

\section{S. Mukhigulashvili}

Institute of Mathematics, Academy of Sciences of the Czech Republic, Žižkova 22, 61662 Brno, Czech Republic, and I. Chavchavadze State University, Faculty of Physics and Mathematics, 32 Chavchavadze St., 0179 Tbilisi, Georgia

E-mail address: mukhig@ipm.cz

\section{B. Půža}

Masaryk University, Faculty of Science, Department of Mathematical Analysis, Janáčkovo nám. 2a, 66295 Brno, Czech Republic

E-mail address: puza@math.muni.cz 